\title{
The Contributions of Orange Fleshed Sweet Potato to Household Vitamin A Intake
}

\author{
Alfred Komakech ${ }^{1,}$, , Okello-Uma Ipolto ${ }^{1}$, Julius Carlos Woodoboma Odongo ${ }^{2}$ \\ ${ }^{1}$ Department of Food Science and Postharvest Technology, Gulu University, Gulu, Uganda \\ ${ }^{2}$ Department of Agronomy, Gulu University, Gulu, Uganda
}

Email address:

komakecha@gmail.com (Alfred K.),okellouma@gmail.com (Okello-Uma I.), juliusodongo@gmail.com (Julius C. W. O.)

${ }^{*}$ Corresponding author

To cite this article:

Alfred Komakech, Okello-Uma Ipolto, Julius Carlos Woodoboma Odongo. The Contributions of Orange Fleshed Sweet Potato to Household Vitamin A Intake. Journal of Food and Nutrition Sciences. Vol. 7, No. 5, 2019, pp. 66-72. doi: 10.11648/j.jfns.20190705.11

Received: September 5, 2019; Accepted: October 4, 2019; Published: October 21, 2019

\begin{abstract}
The prevalence of Vitamin A Deficiency (VAD) remains high in Uganda among children under 5 years and women of reproductive age. The readily bio-available sources of vitamin A such as liver, organ meats and fish oils are too expensive for the majority of the population to consume regularly. Sweet potato is a major staple food in the country and Orange Fleshed Sweet Potato (OFSP) being rich in beta-carotene offers an alternative source of Vitamin A to the low income section of the population. This study was conducted to assess the contributions of OFSP to household vitamin A intake in Gulu district, Northern Uganda. The cross sectional study was conducted over a period of six months in purposively selected four rural sub-counties and the Municipality. A total of 250 randomly selected households participated in the study. Using Food Frequency Questionnaires (FFQ), data on the contributions of different food items to Vitamin A intake was collected. OFSP contributed $68 \%$ of the daily Vitamin A intake at household level. However, OFSP alone is not adequate to meet the vitamin A requirements of all household members. It is therefore important for households to include other sources of Vitamin A in their daily diets to adequately cater for the vitamin A requirements of all its members. To realize the full potential of OFSP or any other food based intervention in addressing malnutrition in a population, it is important to consider other factors such as the health status of individuals, access to health services, sanitation, feeding and care practices since they all play critical roles in determining the overall effect of the intervention on the population.
\end{abstract}

Keywords: Vitamin A Deficiency, Orange Fleshed Sweet Potato, Children Under 5 Years, Women of Reproductive Age

\section{Introduction}

Vitamin A is an essential nutrient required by the body in small amounts for the normal functioning of the visual system, growth and development, maintenance of epithelial cellular integrity, immune function, and reproduction [1]. Vitamin A deficiency (VAD) is of public health significance in the developing world especially among children under 5 years and women of child-bearing age [2]. VAD is normally attributed to consumption of diets that provide too little bioavailable vitamin A to meet the body's physiological needs, children's rapid growth demand for Vitamin A, and frequent infections which lead to ineffective utilization of vitamin A [3]. Globally, VAD is one of the most damaging forms of undernourishment and up to 43 million children under the age of five in Sub-Saharan Africa are vitamin A deficient [4]. The prevalence of VAD among children and women of reproductive age in Uganda was $38 \%$ and $36 \%$, respectively in the year 2011 [5].

Dietary diversification is one of the strategies for combating VAD in a population and it includes all efforts taken to increase Vitamin A or Vitamin A - forming carotenoids in the diet [6]. The dietary sources of vitamin A are: preformed vitamin A which is commonly found in foods of animal origin and pro-vitamin A (i.e., carotenoids) which are found in yellow and orange- fleshed fruits and roots and in dark green leafy vegetables [7]. In developed countries, the pro-vitamin A carotenoids derived from plants provide less than $30 \%$ of daily Vitamin A intake, whereas preformed Vitamin A derived from animal products provides more than $70 \%$ of daily intake [8]. The best sources of preformed 
vitamin A are liver, organ meats and fish oils, but these foods are too expensive for majority of the world's population to eat regularly [6]. In the low income countries, about $82 \%$ of the total vitamin A intake is derived from carotenoids in plants [7]. Bio-fortification is a food-based approach designed to reduce micronutrient malnutrition by increasing the micro-nutrient content of staple foods [9] and promotion of production and consumption of bio-fortified carotenoids-rich foods such as Orange Fleshed Sweet Potato (OFSP) forms an important strategy for increased vitamin A intake in developing countries with limited access to diversified diets, commercially marketed fortified foods or vitamin A supplements.

OFSP varieties are rich in pro-vitamin A and offer a good option in the fight against VAD [10]. Since sweet potato is a major staple food in Uganda [2], OFSP has an advantage over most vegetables in that it can supply significant amounts of vitamin A and energy simultaneously and hence can help to address both VAD and undernutrition [11]. According to [12], "incorporating OFSP into the diet of 3-6 year olds who were marginally deficient in Vitamin A, significantly increased serum retinol concentrations". Two projects: Dissemination of New Agricultural Technologies in Africa (DONATO 2008-2013) and Developing and Delivering of Bio-fortified Crops (DDBC 2012-2016) promoted production and consumption of OFSP in Gulu district, Northern Uganda, focusing on: ensuring access to quality planting materials, creating public awareness on the importance of OFSP in the diet, value addition and access to markets, amongst others. These efforts were geared towards promoting the consumption of OFSP as a strategy to enhance vitamin A intake. No published data has been established in the literature review indicating the contributions of OFSP to household vitamin A intake in Northern Uganda where the aforementioned projects were implemented. This study therefore was designed to assess the contributions of OFSP to household Vitamin A intakes in Gulu district, Northern Uganda. The findings of the study is intended to develop a good understanding of the impacts of the two projects on the community of Northern Uganda.

\section{Method}

The study was conducted over a period of 6 months, starting from October 2015 to March 2016. It was a cross sectional study that covered four out of the twelve rural subcounties and the Municipal council of Gulu district. The study areas were purposively selected because they were considered major areas of production and consumption of OFSP due to their involvement in the implementation of DONATO and DDBC projects. Ethical approval was obtained from Gulu University Research Ethics Committee (GUREC) and permission to carry out the study in the district was obtained from the Chief Administrative Officer.

A total of 250 households were randomly sampled and interviewed from a total of twenty villages in ten parishes within the study area, targeting sweet potato farmers and consumers. The Sample size was derived using a formula;

$$
\mathrm{n}=\frac{\mathrm{t}^{2} \times \mathrm{pq}}{\mathrm{d}^{2}}
$$

Where:

$\mathrm{n}=$ Sample size

$\mathrm{t}=$ value for selected alpha level of 0.025 in each tail $=$ 1.96

$\mathrm{p}=$ the probability of the proportion of households adopting OFSP $\left[\frac{4453}{22,266}=0.2\right]$

(4,453 is the number of households that adopted OFSP and 22,266 is the estimated total number of households in the study area).

$\mathrm{q}=$ the probability of the proportion of households not adopting OFSP

$$
[q=1-p, 1-0.2=0.8]
$$

$\mathrm{d}=$ the acceptable margin of error for proportion being estimated equal to 0.05

$$
n=\frac{\left(1.96^{2}\right) \times(0.2)(0.8)}{0.05^{2}}=245 \approx 250
$$

Of the 250 households, 200 were drawn from the four rural sub-counties and 50 were from the Municipal council. These 50 households were randomly sampled from major sweet potato markets within the Municipality, to capture information from the urban consumers of OFSP. Respondents were fully informed of the purpose of the study and the kind of information required from them before the interviews. Only those that consented to the request were interviewed. Interviews were conducted at appropriate times agreed with the respondents. Confidentiality of the information, views and identity of the respondents was observed. Prior to data collection, assessment of the different food sources from which households potentially derived Vitamin A was made. Plant foods providing 120 retinol equivalents (RE) or 60 retinol activity equivalents (RAE) per $100 \mathrm{~g}$ are considered sources of Vitamin A [13] and this formed the basis for inclusion of food items into the study.

To establish the contributions of the different foods to the overall vitamin A intakes of households, data on the different kinds of vitamin A-rich foods, with their respective quantities, consumed by the households was collected using food frequency questionnaires (FFQ). The FFQ contained 23 different food items which represented the major potential sources of Vitamin A foods of the respondents. Of the twenty three food items included in the FFQ, five food items (Leeks, Broccoli, Cauliflower, Spinach and Cheese) registered no consumption by any of the respondents over the period of the study and were excluded from the analysis. The Vitamin A intake from each food item was computed using Microsoft excel 2013, based on the respondent's frequency of consumption, amount of the food item consumed and the estimated amount of Vitamin A in the quantity of food consumed. The vitamin A intake values from the different food items considered in the study was estimated using [14] National Nutrient database for standard reference. 


\section{Results}

The socio-demographic profile of the respondents is presented in table 1 . It indicates that women $(70 \%)$ were more represented than men $(30 \%)$ and the average age of participants was 38 years. The average household size was 6.8 persons, with up to $87 \%$ of respondent households having at least four members. The majority of the respondent households had children and $76 \%$ of the households had children under five years of age. Compared with the national population census of 2014 , the respondents had $19.2 \%$ more female than the national average (47.6 male and 50.8 female), a larger average household size (6.8 compared to $4.7)$, less people that attained primary level education $(43 \%$ compared to $58.4 \%$ ), but more people who attained ordinary level education (29\% compared to $15.4 \%$ ) [15]. Of the $46 \%$ of the respondents with off-farm income sources, $27 \%$ generated income from small scale businesses, quarry and crafts works while $18 \%$ had salary from formal employment. Only $1 \%$ of the respondents accessed loan as a source of income, from their Village Savings and Loans Associations (VSLA).

Table 1. Socio-demographic characteristics of Respondents.

\begin{tabular}{|c|c|c|c|c|}
\hline Variables & & $\mathbf{N}$ & Percentage (\%) & $\overline{\text { Average }}$ \\
\hline \multirow{2}{*}{ Gender } & Male & 74 & 29.6 & \multirow{5}{*}{38} \\
\hline & Female & 176 & 70.4 & \\
\hline \multirow{3}{*}{ Age } & $19-36$ & 141 & 56.4 & \\
\hline & $37-54$ & 79 & 31.6 & \\
\hline & $55-75$ & 30 & 12 & \\
\hline \multirow{4}{*}{ Household Size } & $1-3$ & 31 & 12.4 & \multirow{4}{*}{6.8} \\
\hline & $4-5$ & 69 & 27.6 & \\
\hline & $6-10$ & 122 & 48.8 & \\
\hline & $11-20$ & 28 & 11.2 & \\
\hline \multirow{4}{*}{ Number of children under 5 years } & 0 & 60 & 24 & \multirow{3}{*}{2.52} \\
\hline & $1-3$ & 184 & 73.6 & \\
\hline & $4-6$ & 6 & 2.4 & \\
\hline & None & 30 & 12 & \\
\hline \multirow{4}{*}{ Education Level } & Primary & 107 & 42.8 & \\
\hline & Ordinary level & 73 & 29.2 & \\
\hline & Advanced level & 22 & 8.8 & \\
\hline & Tertiary & 18 & 7.2 & \\
\hline \multirow{2}{*}{ Sources of Income } & In-farm sources & 135 & 54 & \\
\hline & Off-farm sources & 115 & 46 & \\
\hline \multirow{4}{*}{ Off-farm income sources } & Salary & 45 & 18 & \\
\hline & Small-scale businesses & 27 & 11 & \\
\hline & Quarry, crafts and brick laving works & 40 & 16 & \\
\hline & Loan & 3 & 1 & \\
\hline
\end{tabular}

The average daily intake of the different food items were computed from the FFQ using Microsoft excel version 2013, and the results presented in table 2 .

Table 2. Average daily Household intake of selected Vitamin A sources of food.

\begin{tabular}{lll}
\hline & Food items & Average Daily intake (g) \\
\hline 1 & Orange Fleshed Sweet Potato & 97.9 \\
2 & Pumpkin & 24.7 \\
3 & Carrots & 2.4 \\
4 & Pawpaw & 56.4 \\
5 & Watermelon & 5.3 \\
6 & Kale & 9.2 \\
7 & Cow pea leaves & 70.6 \\
8 & Green beans & 46.5 \\
9 & Tomato & 169.2 \\
10 & Chili pepper & 1.6 \\
11 & Cabbage & 60.8 \\
12 & Egg plant & 70.9 \\
13 & Okra & 80.2 \\
14 & Pumpkin leaves & 45.2 \\
15 & Milk & 32.8 \\
16 & Chicken & 8.9 \\
17 & Eggs & 10 \\
18 & Yogurt & 5.9 \\
\hline
\end{tabular}

The daily Vitamin A intake from the respective quantities of the different food items were computed basing on [14] National Nutrient database for standard reference shown in Table 3 and the results are presented in Table 4. 
Table 3. Vitamin A contents of food stuff.

\begin{tabular}{llll}
\hline \multirow{2}{*}{ Food items } & Vitamin A content per 100g & I.U \\
\cline { 3 - 4 } & & RAE $(\boldsymbol{\mu g})$ & 14187 \\
\hline 1 & Orange Fleshed Sweet Potato (raw) & 709 & 19218 \\
2 & Orange Fleshed Sweet Potato (boiled with skin) & 961 & 15740 \\
3 & Orange Fleshed Sweet Potato (boiled without skin) & 787 & 5753 \\
4 & Pumpkin & 288 & 16706 \\
5 & Carrots (raw) & 835 & 17033 \\
6 & Carrots (cooked) & 852 & 950 \\
7 & Pawpaw & 47 & 569 \\
8 & Watermelon & 28 & 13621 \\
9 & Kale (cooked) & 681 & 576 \\
10 & Cow pea leaves & 29 & 633 \\
11 & Green beans & 32 & 833 \\
12 & Tomato (red ripe - raw) & 42 & 489 \\
13 & Tomato (red ripe -cooked) & 24 & 952 \\
14 & Chili pepper & 48 & 80 \\
15 & Cabbage (cooked) & 4 & 37 \\
16 & Egg plant & 2 & 283 \\
17 & Okra (cooked) & 14 & 1600 \\
18 & Pumpkin leaves & 80 & 162 \\
19 & Milk (whole) & 46 & 597 \\
20 & Chicken (cooked and roasted) & 179 & 787 \\
21 & Eggs (cooked, boiled and fried) & 219 & 6 \\
22 & Yogurt & 2 & \\
\hline
\end{tabular}

Source: USDA National Nutrient Database for standard reference (2016).

$\mathrm{RAE}=$ retinol activity equivalents; 1 retinol activity equivalent $=12-\mu \mathrm{g} \beta$-carotene in food or $1-\mu \mathrm{g}$ purified retinol.

RAE are the unit of measure used by the United States Institute of Medicine (USIOM) to describe the amount of vitamin A contributed to the diet by carotenoids.

I.U = International Unit

Table 4. Computed Daily Vitamin A intake from different food sources.

\begin{tabular}{lllll}
\hline & Food items & $\begin{array}{l}\text { Average Daily food } \\
\text { intake } \mathbf{( g )}\end{array}$ & $\begin{array}{l}\text { Average Daily Vitamin A intake } \\
\text { in } \boldsymbol{\mu g} \text { of RAE }\end{array}$ & $\begin{array}{l}\text { Percentage (\%) of daily Vitamin A } \\
\text { intake by source }\end{array}$ \\
\hline 1 & Orange Fleshed Sweet Potato & 97.9 & 770.5 & 68.0 \\
2 & Pumpkin & 24.7 & 71.1 & 6.3 \\
3 & Carrots & 2.4 & 20.4 & 1.8 \\
4 & Pawpaw & 56.4 & 26.5 & 2.3 \\
5 & Watermelon & 5.3 & 1.5 & 0.1 \\
6 & Kale & 9.2 & 62.7 & 5.5 \\
7 & Cow pea leaves & 70.6 & 20.5 & 1.8 \\
8 & Green beans & 46.5 & 14.9 & 1.3 \\
9 & Tomato & 169.2 & 40.6 & 3.6 \\
10 & Chili pepper & 1.6 & 0.8 & 0.1 \\
11 & Cabbage & 60.8 & 2.4 & 0.2 \\
12 & Egg plant & 70.9 & 1.4 & 0.1 \\
13 & Okra & 80.2 & 11.2 & 1.0 \\
14 & Pumpkin leaves & 45.2 & 36.2 & 3.2 \\
15 & Milk & 32.8 & 15.1 & 1.3 \\
16 & Chicken & 8.9 & 15.9 & 1.4 \\
17 & Eggs & 10 & 21.9 & 1.9 \\
18 & Yogurt & 5.9 & 0.1 & 0.01 \\
Total & & 798.5 & 1133.7 & 100 \\
\hline
\end{tabular}

The results indicated that respondents derived Vitamin A from various sources, both of plant and animal origin. However, the majority of the respondents obtained vitamin A from plant sources. OFSP provided up to $68 \%$ of the average daily vitamin A intake at household level. Foods of animal origin collectively contributed only $4.61 \%$ of the daily Vitamin A intake as shown in figure 1. 


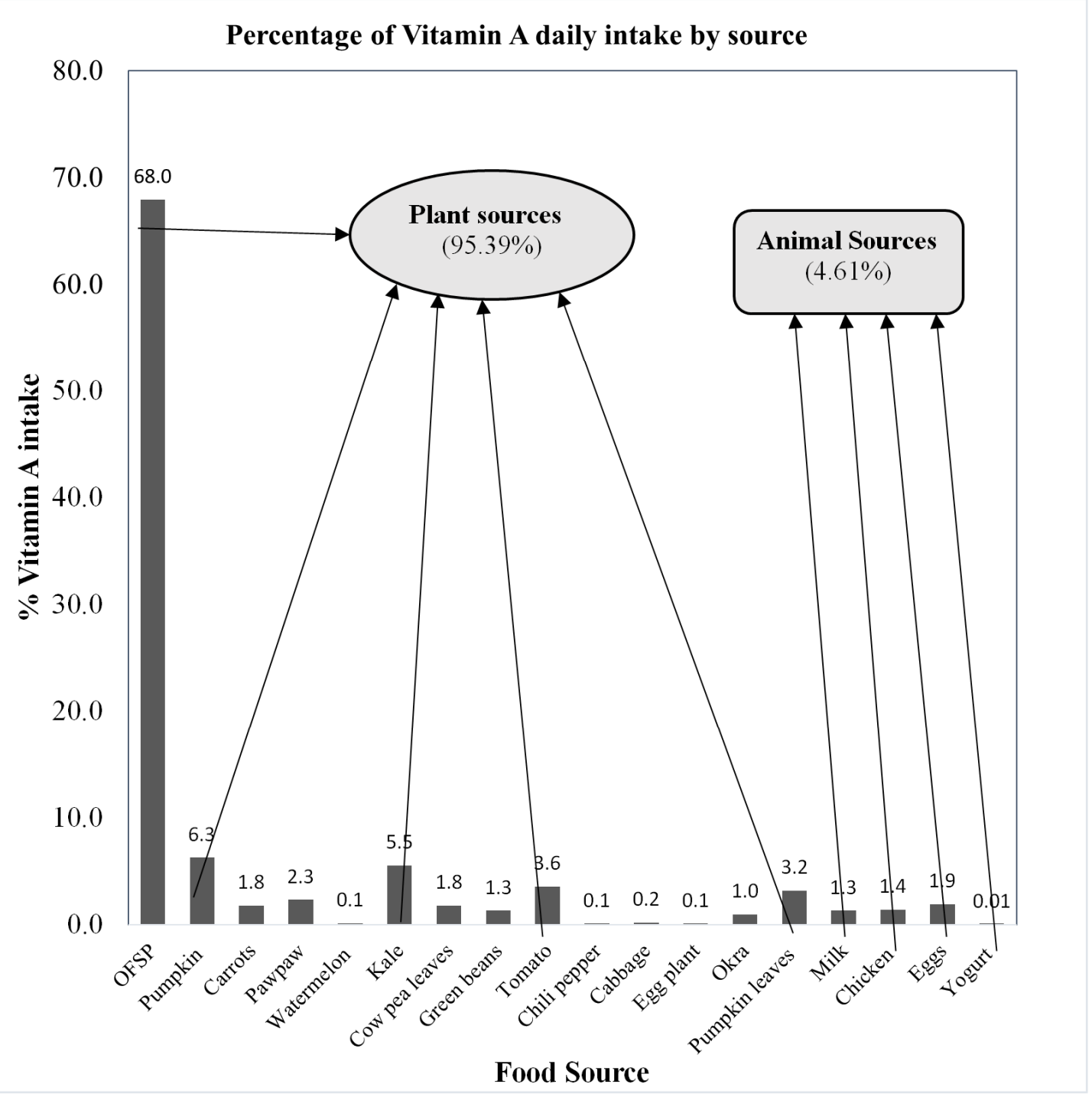

Figure 1. Percentage (\%) of Vitamin A daily intake by source.

The amount and frequency of consumption of the different vitamin A food sources were highly varied. Foods of plant origin were consumed in larger quantities and more frequently compared to foods of animal origin as presented in Table 5.

Table 5. Average quantity and frequency of consumption of selected vitamin A foods.

\begin{tabular}{llll}
\hline Vitamin A food Sources & & Average Quantity of Consumption (g) & Average frequency of Consumption \\
\hline \multirow{4}{*}{ Plant Sources } & OFSP & 173 & $2-4$ times per week \\
& Pumpkin & 149 & $1-3$ times per month \\
& Kale & 94 & Once a week \\
& Tomato & 103 & $2-3$ times per day \\
& Milk & 128 & $1-3$ times per month \\
Animal Sources & Chicken & 126 & $1-3$ times per month \\
& Eggs & 78 & $1-3$ times per month \\
& Yoghurt & $1-3$ times per month \\
\hline
\end{tabular}

\section{Discussion}

The study assessed the contributions of the potential vitamin A sources to the daily vitamin A intake at household level and found that households derived their daily vitamin A mainly from plant sources (Figure 1). The average total daily vitamin A intake from the various sources was estimated at
$1133.7 \mu \mathrm{g}$ retinol activity equivalent (RAE), and according to [6], this level of intake would meet the daily vitamin A intake requirements for all age groups except for lactating mothers. Of this intake (1133.7 $\mu \mathrm{g}$ RAE), animal sources contributed only $4.61 \%$ of the daily vitamin A intake compared to the $95.39 \%$ contributions from plant sources. Foods of plant origin form the major part of the daily diet consumed in poor rural areas because foods of animal origin are less affordable 
on a regular basis [7].

Of the plant sources, OFSP contributed up to $68 \%$ of the total daily vitamin A intake, making it the major source of vitamin A at household level. With an average daily intake of 97.9 grams, OFSP provided households with an estimated daily vitamin A intake of $770.5 \mu \mathrm{g}$ RAE. This amount of daily vitamin A intake from OFSP is adequate to meet the daily requirements for most age groups except for pregnant and lactating mothers who require daily intake of 800 and 1300 $\mu \mathrm{g}$ RAE respectively [6]. Much as this study found OFSP making a huge contribution to household Vitamin A intake, it is important for households to include other sources of vitamin $\mathrm{A}$ as well in their daily diets to adequately cater for the vitamin A requirements of all its members.

The variations in the contributions to vitamin A intake of the different food sources were mainly due to variations in quantity and frequency of consumption and the vitamin A contents of the different food sources. Plant sources were the most consumed in terms of quantity and frequency compared to animal sources (Table 5). Most of the animal sources of vitamin A were consumed only 1 to 3 times per month compared to plant sources which were consumed at least weekly or daily for some food items such as Tomato.

The amount of vitamin $\mathrm{A}$ in a food that is available for absorption by the body is dependent on the food source from which it is derived and the method of food preparation used [11]. Vitamin A derived from animal foods are readily available for absorption but those from plant sources occur in pro-vitamin A forms which upon absorption have to be converted into retinol for the body to make use of it [11]. The bio-accessibility of pro-vitamin $\mathrm{A}$ is the fraction of the provitamin $\mathrm{A}$ in a food that becomes accessible for subsequent absorption by the intestinal mucosa. The size of this fraction of pro-vitamin A from sweet potato depends on sweet potato variety, cooking method and processing methods used [6]. OFSP varieties have beta carotene contents high enough to contribute significantly to the recommended daily vitamin A intake [11]. With respect to cooking, beta carotene in OFSP becomes more bio-available when cooked/boiled and boiling OFSP with the skin minimizes loss of vitamin A [14]. Bioaccessibility vary so that raw OFSP $<$ baked OFSP $<$ steamed/boiled $<$ deep fried OFSP [6]. Inclusion of fat/oil in preparation of OFSP increases the beta carotene bioavailability and [6] observed that even a small amount of fat/oil used to cook OFSP can increase beta carotene bioaccessibility by 2 to 20 folds. Over $90 \%$ of the respondents in this study consumed OFSP in boiled form and only about $8 \%$ prepared OFSP by frying in oil. This implies that a substantial amount of beta carotene is being lost to improper cooking method. It is therefore important for consumers to include fat/oil when preparing OFSP for consumption and in case of boiled roots, it should be boiled with the skin intact, to minimize loss of vitamin A.

Processing methods that involves incorporating OFSP roots with oil/fat enhances its bioavailability and [11] noted that products made from OFSP such as bread, chapatti and cakes were of good quality and acceptable to consumers.
Promoting such value additions therefore contributes to higher consumption and higher bio-availability of vitamin A from OFSP. Processing of OFSP into dried roots was commonly practiced by the respondents and this helped prolonged its shelf life. However, dried OFSP roots stored for more than 4 months experiences dramatic losses in beta carotene content [11]. Although most of the respondents $(80 \%)$ exhausted their dried roots within 3 months, some of them $(20 \%)$ kept their dried roots beyond 4 months and consequently consumed or sold less nutritious OFSP products. Medium to dark orange OFSP varieties should be preferred for processing to lighter orange varieties due to their higher beta carotene contents [11], otherwise all the vitamin A contents could be lost after prolonged storage beyond 4 months.

Consumption and bioavailability of vitamin A from OFSP are important but not adequate to realize the full potential of OFSP in addressing VAD in a population. Other factors such as the health status of individuals, access to health services, sanitation, feeding and care practices play critical roles as well. This is true because VAD has multiple causes. It can be due to inadequate vitamin A intake, or because the sources of vitamin A are not adequately bioavailable, or due to inadequate absorption as a result of disease infections or parasite infestation, or due to increased use by the body as it fights off diseases such as Malaria and Measles [11]. To realize the full potential of a food based intervention in the fight against VAD, focus should take a holistic approach which goes beyond the food based intervention itself to address issues related to food utilization as discussed above (health care, sanitation and feeding practices).

\section{Conclusion}

Consumption of OFSP contributed $68 \%$ of the daily vitamin A intake at household level compared to other food sources, and if complemented with other sources of vitamin A, it can adequately meet the Vitamin A requirements of all household members. However, for effective results, the roots should be cooked in oil/fat, and the dried roots should not be stored for more than four months to minimise loss of Vitamin A. Since majority of the respondents eat mainly boiled OFSP roots, it is recommended that the roots are boiled with the skin intact to minimize Vitamin A losses. Medium to dark orange OFSP varieties should be preferred for processing to lighter orange varieties due to their higher beta carotene contents.

\section{References}

[1] WHO and FAO, "Vitamin and mineral requirements in human nutrition Second edition," World Health Organization, pp. 1$20,2004$.

[2] C. Hotz et al., "Introduction of -Carotene-Rich Orange Sweet Potato in Rural Uganda Resulted in Increased Vitamin A Intakes among Children and Women and Improved Vitamin A Status among Children," Journal of Nutrition, vol. 142, no. 10, pp. 1871-1880, 2012. 
[3] P. J. van Jaarsveld, M. Faber, S. A Tanumihardjo, P. Nestel, C. J. Lombard, and A. J. S. Benadé, "Beta-carotene-rich orangefleshed sweet potato improves the vitamin A status of primary school children assessed with the modified-relative-doseresponse test.," The American journal of clinical nutrition, vol. 81, no. 1, pp. 1080-1087, 2005.

[4] G. Stathers, T., Mkumbira, J., Low, J., Tagwireyi, J., Munyua, H., Mbabu, A. and Mulongo, Orange-fleshed Sweetpotato (OFSP) INVESTMENT IMPLEMENTATION GUIDE. International Potato Center, Nairobi, Kenya, 2015.

[5] Uganda Bureau of Statistics and ICF International Inc, "2011 UGANDA Demography and Health Survey: Addendum to Chapter 11," pp. 1-9, 2012.

[6] B. J. Burri, "Evaluating Sweet Potato as an Intervention Food to Prevent Vitamin A Deficiency," Comprehensive Reviews in Food Science and Food Safety, vol. 10, pp. 118-130, 2011.

[7] WHO, "Global prevalence of vitamin A deficiency in populations at risk 1995-2005," World Health Organization Global Database on Vitamin A Deficiency, vol. 2009, 2009.

[8] G. Tang, "Bioconversion of dietary provitamin A carotenoids to vitamin A in humans," American Journal of Clinical Nutrition, vol. 91, pp. 1468-1473, 2010.

[9] Harvestplus, "This is what we found. Findings from a HarvestPlus Project," 2012.
[10] M. Faber, S. M. Laurie, and P. J. Van Jaarsveld, "Total $\beta$ carotene content of orange sweetpotato cultivated under optimal conditions and at a rural village," African Journal Of Biotechnology, vol. 12, no. 25, pp. 3947-3951, 2013.

[11] J. Low, R. Kapinga, D. Cole, C. Loechl, J. Lynam, and M. Andrade, "CHALLENGE THEME PAPER 3: NUTRITIONAL IMPACT WITH ORANGE-FLESHED SWEETPOTATO (OFSP) Jan Low, Regina Kapinga, Donald Cole, Cornelia Loechl, John Lynam, and Maria Andrade Overall Challenge:," pp. 73-105, 2015.

[12] J. Low, T. Walker, and R. Hijmans, "The potential impact of orange-fleshed sweetpotatoes on vitamin A intake in SubSaharan Africa," The VITAA Project, vitamin A and orangefleshed sweetpotatoes in Sub-Saharan Africa, no. May, pp. 116, 2001.

[13] G. L. Kennedy, T. Ballard, and M. Dop, Guidelines for measuring household and individual dietary diversity. 2010.

[14] N. A. L. United States Dept. of Agriculture, Agricultural Research Service, "National Nutrient Database for Standard Reference,” 2016. [Online]. Available: http://ndb.nal.usda.gov/.

[15] UBOS, "The National Population and Housing Census 2014Main Report, Kampala, Uganda,” pp. 1-209, 2016. 